\title{
Determination of surface stress anisotropy from domain wall fluctuations
}

\author{
H. J. W. Zandvliet and Bene Poelsema \\ Department of Applied Physics and Centre of Materials Research, University of Twente, P.O. Box 217, \\ 7500 AE Enschede, The Netherlands
}

(Received 12 October 1998)

\begin{abstract}
The thermally induced meandering of domain walls between $(2 \times 1)$ and $c(4 \times 2)$ regions on $\mathrm{Ge}(001)$ is analyzed with a scanning tunneling microscope in order to extract the anisotropy of the surface stress tensor. On small length scales the domain walls exhibit random walker behavior, whereas on larger length scales $(>100 \AA)$ due to domain-wall repulsion originating from the anisotropy in the surface stress tensor a deviation from this one-dimensional random-walk behavior is observed. We have determined a value of 0.13 $\pm 0.04 \mathrm{eV} /(1 \times 1)$ cell for the stress anisotropy. [S0163-1829(99)02411-X]
\end{abstract}

Surface stress plays a major role in many surface phenomena. For instance, surface stress can stabilize the surface atomic structure or control the growth mode of heteroepitaxial thin films. Recent studies of the lattice mismatched $\mathrm{Ge} / \mathrm{Si}(001)$ system have shown a wealth of fascinating phenomena, such as the formation of $(2 \times n)$ reconstructions, ${ }^{1}$ reversal of step-edge roughness, ${ }^{2}$ and the formation of threedimensional islands, ${ }^{3}$ in which surface stress plays the key role. More recently, we have addressed the influence that surface stress anisotropy can have on the structure of clean semiconductor group-IV (001) surfaces. ${ }^{4}$ It is well established that at room temperature the technologically important $\mathrm{Si}(001)$ and $\mathrm{Ge}(001)$ surfaces exhibit a $(2 \times 1)$ reconstruction: surface atoms dimerize, eliminating one dangling bond per atom (at the expense of the development of an anisotropic surface stress tensor) to lower the surface-free energy. This dimerization was first proposed by Schlier and Farnsworth $^{5}$ nearly 40 years ago and was imaged on an atomic scale in real space by scanning tunneling microscopy (STM) in $1985 .{ }^{6}$ Recently, we have shown that this generally accepted view on the ground state (at room temperature) of the semiconductor group-IV (001) surfaces needs to be adjusted. The state with the lowest surface-free energy at room temperature of these surfaces is not a simple $(2 \times 1)$ reconstruction as generally accepted by the physical community ${ }^{5,6}$ (and taught to students in textbooks on surface science). Instead, it consists of an ordered pattern of alternating $c(4 \times 2)$ and $(2 \times 1)$ domains [also, in the $c(4 \times 2)$ reconstruction the dimers are the elementary building blocks: the dimers are buckled in an antiferromagnetic ordering along a dimer row, whereas adjacent dimer rows are out of phase with respect to the buckling registry]. In retrospect, the occurrence of this structure is not strange at all but can in fact be explained in the framework of a well-established strain relaxation theory. ${ }^{7}$ The reason why this structure has not been observed before so far is that it requires extremely clean and defect-free surfaces. For the $\mathrm{Si}(001)$ surface it is inherently difficult to prepare clean surfaces with defect-free densities low enough for this pattern to develop. For the Ge(001) surface, however, this requirement can be met by extremely careful preparation procedures. Interestingly, in the particular case where the domain-wall free energy vanishes, which ap- plies reasonable well to the $\mathrm{Ge}(001)$ case, the size of the domain pattern does not depend on the actual magnitude of the surface stress anisotropy. Due to the surface stress anisotropy, domain walls exhibit a force monopole. These force monopoles repel each other resulting in a reduction of the thermally induced meandering of the domain walls on larger length scales. Here we demonstrate that by analyzing the thermally induced meandering of the domain walls we are able to extract the surface stress anisotropy experimentally. The method we apply here is generally applicable to other surface systems where the domain pattern is stabilized by long-range interactions, such as electrostatic or magnetic interactions.

The experiments have been conducted in an ultrahigh vacuum (UHV) chamber (base pressure $1 \times 10^{-10} \mathrm{mbar}$ ) equipped with a scanning tunneling microscope. The nominally flat $n$-type $\mathrm{Ge}(001)$ samples were cleaned by cycles of sputtering with $\mathrm{Ar}^{+}$ions and annealing at $1100 \mathrm{~K}$. The samples are either rapidly quenched by switching of the power supply or slowly cooled $(1 \mathrm{~K} / \mathrm{s})$ to room temperature. Samples repeatedly cleaned in this way typically contain $0.02-0.5 \%$ surface defects.

Figure 1 shows a dimer resolved STM image of a clean and nearly defect-free nominally flat intrinsic $\mathrm{Ge}(001)$ surface revealing the ordered $(2 \times 1) / c(4 \times 2)$ domain pattern. First-principles calculations of the surface stress for the $(2 \times 1)$ and $c(4 \times 2)$ dimer reconstructed (001) surfaces show that the tensile stress component along the dimer bond is about one tenth of an $\mathrm{eV} /(1 \times 1)$ cell larger for the $c(4 \times 2)$ reconstruction than for the $(2 \times 1)$ reconstruction. ${ }^{8,9}$ The stress component perpendicular to the dimer bond is compressive for both the $(2 \times 1)$ and $c(4 \times 2)$ reconstructions. Consider for the sake of simplicity a quasi-onedimensional ordered domain structure consisting of alternating $(2 \times 1)$ and $c(4 \times 2)$ domains. At each domain wall between a $(2 \times 1)$ and $c(4 \times 2)$ domain a force density, equal to the anisotropy in the stress component, is present. Such a configuration will result in strain relaxation, which decays logarithmically with the domain width. ${ }^{7}$ Irrespective of the exact value of domain-wall formation energy one can always find a critical domain width where the surface-free energy exhibits a minimum. ${ }^{7}$ If we consider a striped domain phase 


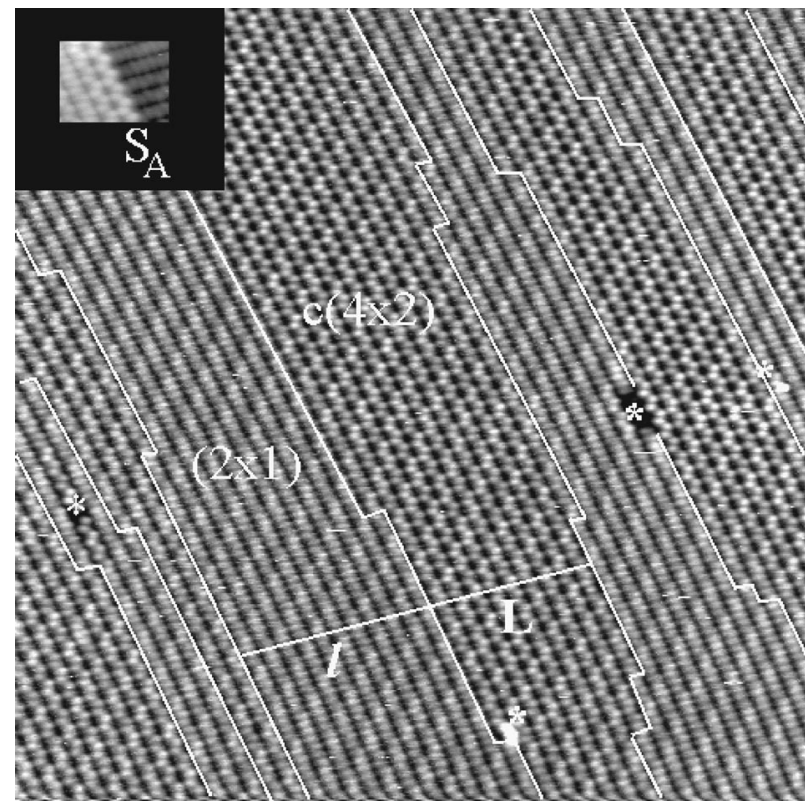

FIG. 1. STM image of a clean $\mathrm{Ge}(001)-(2 \times 1) / c(4 \times 2)$ surface. Scan area $400 \times 400 \AA^{2}$. Sample bias is $-1.6 \mathrm{~V}$ and tunneling current is $1 \mathrm{nA}$. Asterisks mark surface defects, such as missing dimer clusters and adsorbates. Inset: Buckling registry near a monatomic $S_{A}$ step edge.

consisting of $(2 \times 1)$ and $c(4 \times 2)$ domains of widths $l$ and $L$, with free energies per unit area of $\gamma_{2 \times 1}$ and $\gamma_{c(4 \times 2)}$, respectively. The formation-free energy of the domain wall per unit length between the two domains is denoted by $F_{\text {wall }}$, and the anisotropy of the surface stress component along the dimer bond is $\sigma_{c(4 \times 2)}-\sigma_{2 \times 1}$. The population of the two domains are $p$ and $(1-p)$, respectively. The free energy per unit area, $F /(l+L)$, is represented by ${ }^{4,10}$

$$
\begin{aligned}
\frac{F}{l+L}= & p \gamma_{2 \times 1}+(1-p) \gamma_{c(4 \times 2)}+\frac{2 F_{\text {wall }}}{l+L} \\
& -\frac{2 C}{(l+L)} \ln \left[\frac{l+L}{2 \pi a_{0}} \sin (\pi p)\right]
\end{aligned}
$$

with $C=(1-\nu)\left[\sigma_{c(4 \times 2)}-\sigma_{2 \times 1}\right]^{2} / 2 \pi \mu$.

Here, the first two terms refer to the free energy per unit area of the two different domains; the third term to the formation of the domain walls, and the fourth term to the strain relaxation energy, where $a_{0}$ is a microscopic cutoff length (we assume here that the surface lattice constant, $=4 \AA$, is the microscopic cutoff length). $\mu$ and $\nu$ are the bulk modulus and Poisson's ratio of the medium, respectively $[(1-\nu) / 2 \pi \mu$ $\left.\approx 0.01 a^{3} / \mathrm{eV}\right]$. The width, $(l+L)_{c}$, for which the free energy per unit area is at its minimum is

$$
(l+L)_{c}=\frac{2 \pi a_{0}}{\sin (\pi p)} e^{\left(F_{\mathrm{wall}} / C+1\right)} .
$$

At room temperature the $(2 \times 1)$ and $c(4 \times 2)$ domains are about equally populated, i.e., $p=\frac{1}{2}$, and if we assume that $F_{\text {wall }} \ll C$ one finds a critical domain width of about $70 \AA$. The experimentally determined domain width is about $80-90$ $\AA$, which indicates that $F_{\text {wall }}$ is indeed significantly smaller than $C .^{4}$ A similar domain pattern formation along the dimer row direction is to be expected as well; however, the domain wall energy in this case is quite substantial ${ }^{4}$ resulting in extremely large domains $\left[(l+L)_{c}\right.$ depends exponentially on $\left.F_{\text {wall }}\right]$.

The inset of Fig. 1 shows the ordering of the dimers near a monatomic $S_{A}$ step edge. The $A$-type upper terrace nearly always terminates with a $c(4 \times 2)$ domain. This can easily be understood in terms of strain relaxation, i.e., minimalization of the free energy per unit area. The largest force monopole will be found at the monatomic $A$-type step edge if the upper terrace terminates with a $c(4 \times 2)$ domain, because the tensile stress component along the dimer is larger for the $c(4 \times 2)$ reconstruction than for the $(2 \times 1)$ reconstruction.

In order to analyze the spatial fluctuations of the domain walls, we introduce a $x-y$ coordinate system with the $x$ direction taken to be the mean running direction of the domain wall. The spatial fluctuations of the domain walls can be characterized by the mean-square displacement as a function of distance $x:\left\langle[y(x)-y(0)]^{2}\right\rangle$. An isolated domain wall behaves as a random walker, and therefore, the mean-square displacement increases linearly with $x^{11}$

$$
\left\langle[y(x)-y(0)]^{2}\right\rangle=\left\langle k^{2}\right\rangle \frac{x}{a_{\|}},
$$

where $\left\langle k^{2}\right\rangle$ is the domain wall diffusivity

$$
\left\langle k^{2}\right\rangle=\frac{a_{\perp}^{2} \sum_{-\infty}^{\infty} k^{2} e^{-\epsilon(k) / k_{b} T}}{\sum_{-\infty}^{\infty} e^{-\epsilon(k) / k_{b} T}} .
$$

To extract $\left\langle k^{2}\right\rangle$ from microscopic interactions, we need to know $\epsilon(k)$, the energy of a kink of length $2 k a_{\perp}(k=\ldots$, $-2,-1,0,1,2, \ldots)$. In principle there are two different types of kinks, which are called positive or negative, corresponding to a "protrusion" and an "indentation," respectively, that can be distinguished. The symbol $n_{+(-)}$is used to denote the probability that at a given position in the domain wall there is a protrusion (indentation) of length $2 a_{\perp}$ in a direction perpendicular to the domain wall; $n_{0}$ is the probability that at a given position in the domain wall there is no kink of any kind. According to the Burton, Cabrera, and Frank (BCF) theory, ${ }^{12}$ the following thermodynamic relation can be derived for a domain wall: $n_{+} n_{-} n_{0}^{-2}=\exp$ $\left(-2 \varepsilon / k_{b} T\right)$, where $\varepsilon$ is kink creation energy for a single kink. From a statistical analysis of several STM images we have found a single kink creation energy of $\varepsilon=0.095 \mathrm{eV} / 2 a_{\perp}$ $\left(a_{\perp}=a_{\|}=a=4 \AA\right)$.

Consider now the more realistic case of a meandering domain wall trapped between two neighboring walls. Repulsive entropic and energetic interactions between the walls will tend to limit the amount of meandering by making closely spaced domain walls unfavorable. When the walls begin to collide with each other, i.e., when the mean-square displacement becomes a significant fraction of the averaged domain-wall spacing $(L)$, deviations from linear, "diffusive" behavior must occur. Before continuing, it would be very illustrative to give an estimate for the entropic repulsion. Let us, for the sake of simplicity, consider a meandering domain wall trapped between two hard walls with the only restriction 
being that domain walls are not allowed to cross. Each collision reduces the entropy with about $k_{b} \ln (2)$. The typical spacing between successive domain-wall collisions is $L^{2} /\left\langle k^{2}\right\rangle$. Hence, the free-energy increase per unit length along the domain wall is about $k_{b} T\left\langle k^{2}\right\rangle \ln (2) / L^{2}$. Using our experimental data of $\left\langle k^{2}\right\rangle=0.219 a_{\perp}^{2}, L=10 a_{\perp_{0}}$ and $T$ $=300 \mathrm{~K}$ gives a free-energy increase of $0.04 \mathrm{meV}$ per unit domain-wall length $a_{\|}$. However, we can get rid of this entropic domain-wall repulsion if we randomize the labeling of the domain walls upon a collision. However, because we only observed a limited number of collisions we have applied another scheme: after each collision we have considered both possibilities (i.e., crossing and reflection of the walls) and have determined the averaged contribution to the mean-square displacement. The other repulsive interaction, which arises here due to the existence of an anisotropic surface stress, results also in a flattening out of the mean-square displacement. The simplest scheme to account for this is to take the continuum limit in the $x$ direction, which reduces the problem to solving the one-dimensional Schrödinger equation for a harmonic oscillator [if the strain relaxation term $C \ln (L)$ is expanded for small $\delta L$ around the minimumenergy configuration, the leading harmonic term $\left.\sim C(\delta L)^{2} / L^{2}\right]$. Using path integral arguments one finally finds ${ }^{13}$

$$
\begin{aligned}
\left\langle[y(x)-y(0)]^{2}\right\rangle \approx & \sqrt{\frac{k_{b} T\left\langle k^{2}\right\rangle L^{2}}{2 C}} \\
& \times\left[1-\exp \left(-x \sqrt{\frac{2 C\left\langle k^{2}\right\rangle}{k_{b} T L^{2}}}\right)\right] .
\end{aligned}
$$

In Fig. 2 a plot of the experimentally determined meansquare displacement versus $x$ is displayed. Initially the meansquare displacement exhibits one-dimensional random-walk behavior, i.e., the mean-square displacement scales linear with $x$. At larger length scales the influence of the repulsive interaction between the domain walls shows up, resulting in a slight reduction of the mean-square displacement. Surface defects such as adsorbates and missing dimer clusters seem to induce, at least locally, $c(4 \times 2)$ or $p(2 \times 2)$ buckling. Therefore, we have only determined the mean-square displacement of domain walls that are completely free of those surface defects. Two additional complications are that in most cases it is hard to determine the exact position of a kink (usually the buckling decays over about ten dimer positions) and that domain walls exhibit some thermal motion at room temperature. In Fig. 2 a slight deviation from the ideal onedimensional random walker (with $\left\langle k^{2}\right\rangle \equiv\left\langle[y(1)-y(0)]^{2}\right\rangle$ is

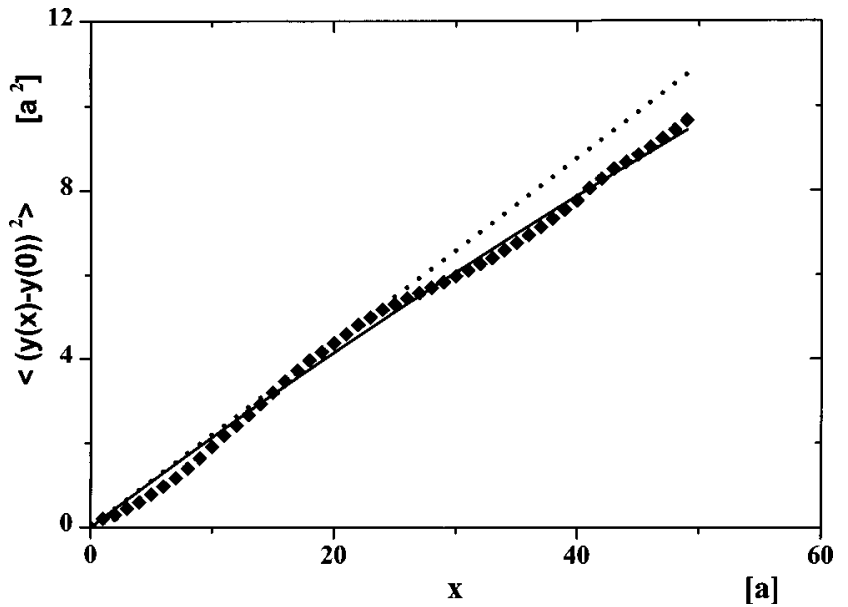

FIG. 2. Mean-square displacement $\left\langle[y(x)-y(0)]^{2}\right\rangle$ versus position $x$ measured along the domain wall in units of $a^{2}$ and $a$, respectively. Diamonds: experimental data points (the statistical error for $\left\langle[y(49)-y(0)]^{2}\right\rangle$ is estimated to be $0.2 a^{2}$ using the procedure outlined in Ref. 14). Dotted line: one-dimensional random walker. Solid line: Eq. (5) with $\sigma_{c(4 \times 2)}-\sigma_{2 \times 1}=0.13 \mathrm{eV} / a^{2}$.

$0.219 a_{\perp}^{2}$ ) can clearly be observed for larger length scales $(>100 \AA)$. We have also depicted the theoretical curve [Eq. (5)] using a surface stress anisotropy of $\sigma_{c(4 \times 2)}-\sigma_{2 \times 1}$ $=0.13 \mathrm{eV} / a^{2}$ (the error in the only free parameter, $\sigma_{c(4 \times 2)}$ $-\sigma_{2 \times 1}$, is estimated to be about $\left.0.04 \mathrm{eV} / a^{2}\right)$. We suggest that the slight oscillation with a period of about $25 a_{\|}(=100$ $\AA$ ) might be due to the fact that neighboring kinks tend to repel each other. Using first-principles calculations García and Northrup ${ }^{8}$ found an anisotropy of $0.1 \mathrm{eV} / a^{2}$ for the closely related $\mathrm{Si}(001)$ surface. Although similar calculations for the $\mathrm{Ge}(001)$ surface are not presently available, we assume that a similar anisotropy in the surface stress exists.

In conclusion, the existence of anisotropy of the surface stress component along the dimer bond for $(2 \times 1)$ and $c(4 \times 2)$ dimers, respectively, is responsible for the spontaneous formation of a striped $(2 \times 1) / c(4 \times 2)$ domain pattern on clean $\mathrm{Ge}(001)$. The periodicity of this striped domain pattern is, for the case of a vanishing domain-wall free energy, independent of the exact strength of the surface stress anisotropy, $\sigma_{c(4 \times 2)}-\sigma_{2 \times 1}$. However, the thermally induced meandering of the domain walls is sensitive for the exact strength of the surface stress anisotropy. By careful analysis of the mean-square displacement we have extracted a value of $0.13 \pm 0.04 \mathrm{eV} /(1 \times 1)$ cell for $\sigma_{c(4 \times 2)}-\sigma_{2 \times 1}$, which agrees well with the theoretically determined values for the closely related $\mathrm{Si}(001)$ surface.
${ }^{1}$ X. Chen, F. Wu, Z. Zhang, and M. G. Lagally, Phys. Rev. Lett. 73, 850 (1994)

${ }^{2}$ F. Wu, X. Chen, Z. Zhang, and M. G. Lagally, Phys. Rev. Lett. 74, 574 (1995).

${ }^{3}$ F. Wu and M. G. Lagally, Phys. Rev. Lett. 75, 2534 (1995).

${ }^{4}$ H. J. W. Zandvliet, B. S. Swartzentruber, W. Wulfhekel, B. J. Hattink, and B. Poelsema, Phys. Rev. B 57, R6803 (1998).

${ }^{5}$ R. Schlier and H. Farnsworth, J. Chem. Phys. 30, 917 (1959).
${ }^{6}$ R. M. Tromp, R. J. Hamers, and J. E. Demuth, Phys. Rev. Lett. 55, 1303 (1985).

${ }^{7}$ O. L. Alerhand, D. Vanderbilt, R. D. Meade, and J. D. Joannopoulos, Phys. Rev. Lett. 61, 1973 (1988).

${ }^{8}$ A. García and J. E. Northrup, Phys. Rev. B 48, 17350 (1993).

${ }^{9}$ J. Da̧browski, E. Pehlke, and M. Scheffler, Phys. Rev. B 49, 4790 (1994).

${ }^{10}$ In Ref. 4 we have used the domain-wall formation energy $E_{\text {wall }}$ 
rather than the domain-wall free energy $F_{\text {wall }}$. Using the free energy here is, however, more appropriate. If we assume that a kink with a length of $k$ units costs energy $k \varepsilon$ then the partition function $Z$ becomes

$$
Z=e^{-E_{\text {wall }} / k T}\left(1+2 \sum_{k=1}^{\infty} e^{-k \varepsilon / k T}\right)
$$

and the domain-wall free energy (per unit length $a$ )

$$
F_{\text {wall }}=-k T \ln Z=E_{\text {wall }}-k T \ln \left(\frac{1+e^{-\varepsilon / k T}}{1-e^{-\varepsilon / k T}}\right)=E_{\text {wall }}-1.3 \mathrm{meV} .
$$

Using $(l+L)_{c}=90 \AA$ and $\sigma_{c(4 \times 2)}-\sigma_{2 \times 1}=0.13 \mathrm{eV} / a^{2}$ we find that the domain-wall energy is $1.34 \mathrm{meV} / \mathrm{a}$ ( $F_{\text {wall }}$ $\approx 0.04 \mathrm{meV} / \mathrm{a}$ ).

${ }^{11}$ N. C. Bartelt, T. L. Einstein, and E. D. Williams, Surf. Sci. 276, 308 (1992).

${ }^{12}$ W. K. Burton, N. Cabrera, and F. C. Frank, Philos. Trans. R. Soc. London, Ser. A 243, 299 (1951).

${ }^{13}$ H. J. W. Zandvliet, H. K. Louwsma, P. E. Hegeman, and B. Poelsema, Phys. Rev. Lett. 75, 3890 (1995).

${ }^{14}$ G. Ayrault and G. Ehrlich, J. Chem. Phys. 60, 281 (1974). 